SANDIA REPORT

SAND98-0702 • UC-706

Unlimited Release

Printed March 1998
FIECENE

\title{
Development of Laser Cutting/Welding Depth Control Using Laser Spectroscopy and Open Architecture Control of a Robotic System
}

DISTRIBUTION OF THIS DOCUMENT IS UNLMITED

G. Randall McKee, Lilita Meirans, Dan J. Schmitt, Dan Small, Peter A. Watterberg, Steven Siegel

\section{Prepared by}

Sandia National Laboratories

Albuquerque, New Mexico 87185 and Livermore, California 94550

Sandia is a multiprogram laboratory operated by Sandia Corporation,

a Lockheed Martin Company, for the United States Department of

Energy under Contract DE-AC04-94AL85000.

Approved for public release; further dissemination unlimited.

\section{G. Sandia National Laboratories}


Issued by Sandia National Laboratories, operated for the United States Department of Energy by Sandia Corporation.

NOTICE: This report was prepared as an account of work sponsored by an agency of the United States Government. Neither the United States Government nor any agency thereof, nor any of their employees, nor any of their contractors, subcontractors, or their employees, makes any warranty, express or implied, or assumes any legal liability or responsibility for the accuracy, completeness, or usefulness of any information, apparatus, product, or process disclosed, or represents that its use would not infringe privately owned rights. Reference herein to any specific commercial product, process, or service by trade name, trademark, manufacturer, or otherwise, does not necessarily constitute or imply its endorsement, recommendation, or favoring by the United States Government, any agency thereof, or any of their contractors or subcontractors. The views and opinions expressed herein do not necessarily state or reflect those of the United States Government, any agency thereof, or any of their contractors.

Printed in the United States of America. This report has been reproduced directly from the best available copy.

Available to DOE and DOE contractors from

Office of Scientific and Technical Information

P.O. Box 62

Oak Ridge, TN 37831

Prices available from (615) 576-8401, FTS 626-8401

Available to the public from

National Technical Information Service

U.S. Department of Commerce

5285 Port Royal Rd

Springfield, VA 22161

NTIS price codes

Printed copy: A03

Microfiche copy: A01

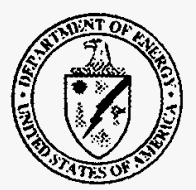


SAND98-0702

Distribution

Unlimited Release

Category UC-706

Printed March 1998

\title{
Development of Laser Cutting/Welding Depth Control Using Laser Spectroscopy and Open Architecture Control of a Robotic System
}

\author{
G. Randall McKee \\ Applied Systems II Department \\ Lilita Meirans \\ Applied Systems I Department \\ Dan J. Schmitt \\ Intelligent Systems Sensors and Controls Department \\ Dan Small \\ Engineering and Manufacturing Software Department \\ Peter A. Watterberg \\ Intelligent Systems Principles Department \\ Sandia National Laboratories \\ P.O. Box 5800 \\ Albuquerque, NM 87185-1007 \\ Steven Siegel \\ PaR Systems, Inc. \\ Shoreview, MN 55126
}

\begin{abstract}
This report documents the work accomplished under the one year funded Laboratory Directed Research and Development (LDRD) titled, "Development of Laser Cutting/Welding Depth Control Using Laser Spectroscopy and Open Architecture Control of a Robotic System." This project was driven by the need to identify and provide unique, state-of-the-art solutions to the robotic path planning and precision motion execution problems that face automated processes such as welding and cutting using lasers. The initial LDRD proposal was for a full three-year program with a schedule that would create a precision robotic platform capable of providing path planning and precision motion execution using sensor and graphical programming technologies as the first years milestone. Milestones for year two were centered in developing and deploying sensor technologies that support welding and cutting. And year three milestones included the integration of any developed sensors onto the robotic platform under software control to achieve autonomous control of laser welding and cutting processes. The work performed was directed at the goal of establishing a precision robotics platform with the capability to integrate graphical programming, CAD model based path planning, and motion execution under real-time sensor based control. This report covers the progress made toward that goal during the one year of funding.
\end{abstract}




\section{Acknowledgment}

This LDRD project was a strong team effort and the acknowledgment of the entire team in this report is required. The development team was led by Dan Schmitt who provided sensor based control guidance as well as project management and scheduling. Lilita Meirans and Peter Watterberg provided the path planning and robotic configuration software technologies and software integration. Dan Small provided the graphical programming technology based in his work in Sancho and software integration. Jon Bryan performed the capacitive sensor development and integration tasks and sensor systems testing. And finally, John Webb provided the robotic hardware implementation and integration tasks for the project. PaR Systems, Inc. provided extensive input and effort in the robotic platform through Steven Siegel and Rinto Dasuki. 


\section{Table of Contents}

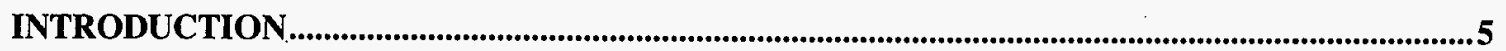

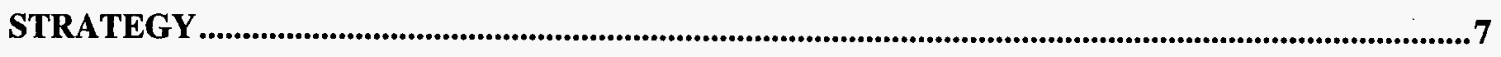

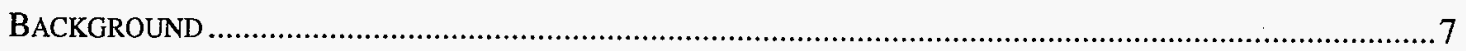

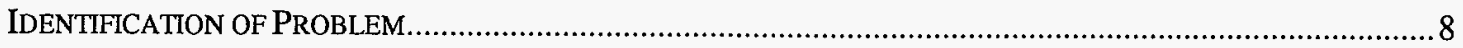

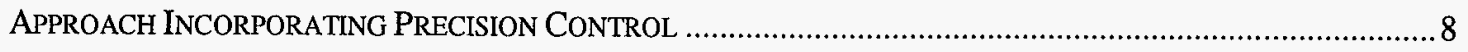

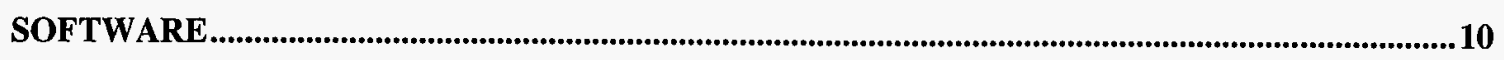

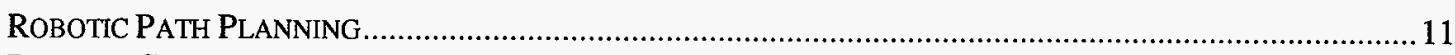

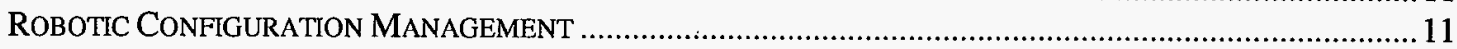

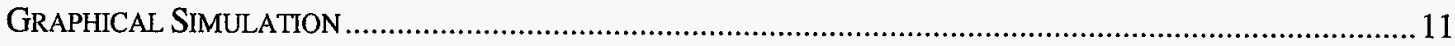

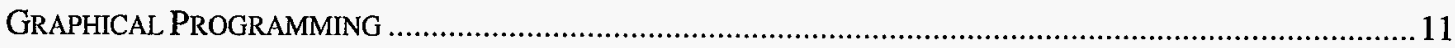

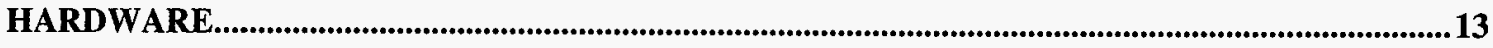

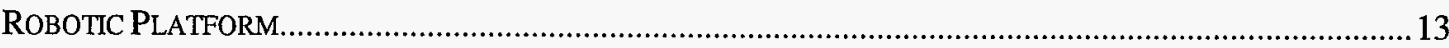

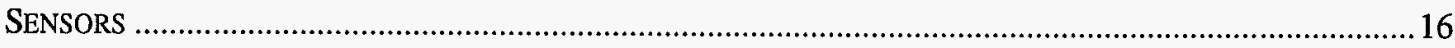

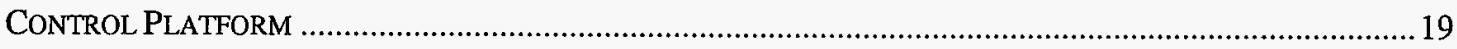

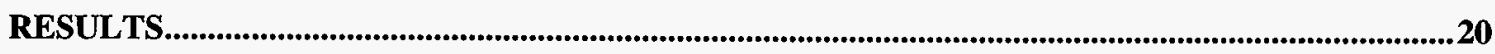

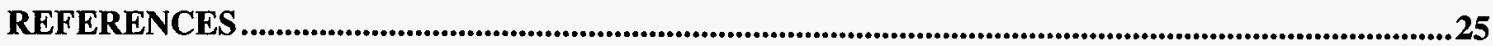




\section{Table of Figures}

Figure 1. Logic Diagram of ProJect SofTWARE Control ..........................................................10

Figure 2. HARdware Diagram wIth Communication TeChNOLOGY IDENTIFIED ............................13

FIGURE 3. PAR RoBOTIC SYSTEM ACCURACY BY AXIS .............................................................................14

Figure 4. Photo of the PaR Xr-225 Precision Robotic Gantry Platform...................................15

Figure 5. CAPACITIVE Standoff SEnSOR ON Robotic Platform .......................................................17

FIGURE 6. DIAGRAM OF STANDOFF SENSOR ...........................................................................................18

Figure 7. SimULATION OF ROBOTIC PLATFORM AND WORKCELL ................................................................20

Figure 8. Simulation of Planar Cut Through Cylinder To Establish Path................................21

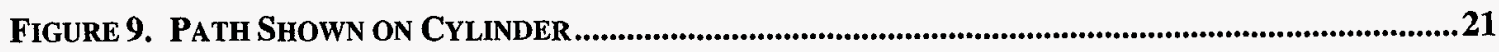

FIGURE 10. SIMULATION SHOWING A COLLISION HIGHLIGHTED ..................................................................22

FiguRe 11. Simulation OF Robot ExeCUTING PlanNEd PATH .....................................................................23 


\section{Development of Laser Cutting/Welding Depth Control Using Laser Spectroscopy and Open Architecture Control of a Robotic System}

\section{Introduction}

The purpose of this project was the research and development of real-time sensor based controlled robotic laser cutting and welding technologies that support defense programs in weapons manufacture and stockpile surveillance. Certified welding processes are required for many NQA-1 weapons manufacturing programs and as such are very interesting to the DOE Weapons Complex. These welding processes are typically performed manually due to the complexity of the process itself, and are accomplished by a welder with a high degree of eye-hand coordination coupled with extensive process knowledge. Validation of the process is accomplished using extensive welder certification programs, Non Destructive Analysis (NDA) of the components welded, and functional testing (usually destructive) of assemblies. These are very expensive processes that add burden to budgets that are shrinking. This, combined with the Comprehensive Test Ban Treaty that limits testing, is driving design and manufacturing agencies in the Weapons Complex to look at alternative methods of validating processes. Autonomous processes that are performed to specification are a means to achieve this validation requirement, and the DOE is currently looking at this in their advanced manufacturing effort programs.

A primary goal of this project was to achieve incremental steps toward developing autonomous welding processes that will ultimately lead to a certified welding capability using robotics, and lasers or conventional Tungsten Inert Gas (TIG) systems.

The technology development in this LDRD project was centered in the utilization of laserinduced spectroscopy and capacitive standoff sensing as real-time sensors for precision motion and process control of laser welding and cutting. A principle goal of this project was the integration of sensors onto a high precision, five and six degree of freedom robotics platform capable of delivering laser processes such as welding and cutting.

The initial research and development schedule of this LDRD project had laser-induced spectroscopy targeted as a primary technology for the real-time sensor for monitoring and controlling a laser cutting application. This process specific sensor development effort was being performed at Los Alamos National Laboratory's Nuclear Materials Technology Division, (NMT) and specifically in the Advanced Technology Group ${ }^{1}$. Also targeted as a primary sensor technology was capacitive standoff sensing developed in Sandia National Laboratories Intelligent Systems and Robotics Center ${ }^{2}$. The capacitive sensor was chosen to be the first sensor implemented onto the platform when it became evident that the spectroscopic sensor required 18 months to move it from the development stage to a packaged sensor. 
The majority of the work performed was in the development of a control scheme on the robotic platform that allowed the sensor integration. This work included bringing robotic path planning using $\mathrm{CAD}$ models, robotic configuration management, graphical simulation of the robotic system, and graphical programming all onto a single control platform that directed the precision motion of a gantry robot. Significant effort was directed at creating an effective precision motion planning and execution capability on the robotic platform. This was required to assure that the development and test of the sensors selected could be performed under optimal conditions. 


\section{Strategy}

This section describes the problems of autonomous control of non-contact processes such as laser cutting and welding and the approach taken by the project to make incremental steps towards a composite solution.

\section{Background}

In manufacturing environments where a welding or cutting process is required to be certified to high quality standards, the process will typically be performed manually by a certified welder. This welder possesses a high degree of eye-hand coordination with many years of process knowledge and experience. As he executes the process he uses this eyehand coordination and process experience to manage the welding operation to assure that the correct penetration and amount of filler material is incorporated. The welder in fact is essentially providing real-time control of the motion process under constantly changing parameters. As the material distorts and moves, he alters the point of process location and tracking speed to meet the penetration requirements established to meet predetermined quality guidelines. The welder knowledge base is created by his understanding and knowledge of three fundamental process parameters. They are welding/cutting power input or control, point of process location, and process speed and feed. Considering these inputs to the welding process, the argument can be made that automation is a next step, as all three of these parameters are easily automated and controlled separately. In fact, great numbers of automated and robotic welding processes are deployed in industry. These processes are automated but not autonomous, the difference being the level of process parameter control performed by the system itself. An automated process has a pre-set path, power level and part presentation. It executes the process exactly the same way every time it is performed. If a part distorts excessively, or a transformer in the power supply fails and lowers the welding current, the process controller does not effect a repair while it is executing. It typically finishes the process oblivious to the distortion or stops the process due to a flag noting an out of specification parameter. Both actions result in a failed part run or scrap material. In an autonomous process the delivery platform incorporates sensors, fixtures, path planning and modification capability, and precision motion delivery to perform the process under changing conditions. In the case mentioned above, as material distorts, the focal point position may be altered to assure correct weld penetration, or as the power supply failed and current dropped, the process speed might be slowed to assure the average energy input for that weld schedule is maintained.

For this autonomous execution of non-contact processes such as laser cutting and welding, a fundamental need is the ability to control precision placement of the point of process in the work field in real-time. This need is directed by the process itself, as cutting and welding are thermal based operations that result in the distortion of the materials being processed. This distortion results in poor process quality when penetration becomes variable, or seam/cutting line tracking becomes unpredictable. Material movement due to this thermal stressing results in an ever changing environment that a standard automated system cannot be pre-programmed to deal with. 


\section{Identification of Problem}

An automated process delivery platform typically is comprised of a 6 degree of freedom robotic arm or gantry robot, a laser or TIG welder and a computer control system. The control of this automated process has fundamentally been approached using standard machine tool code such as RS-274. The utility of these codes is very well understood, but they are extremely limited in their ability to incorporate real-time sensor input for process control.

As stated, the defining issue of autonomous control of welding and cutting processes is the ability to precisely control the position, power, and speed of the point of process in realtime. The point of process is defined in this report as the laser focal point of a laser cutting/welding application or the tungsten tip of a Tungsten Inert Gas (TIG) welding process. It is this requirement that creates the opportunity for the research and development effort undertaken in this project.

The ability to precisely control the point of process parameters requires a supervisory control platform that can process sensor input and effect parameter changes at speeds that support the process being executed. In this program, laser cutting and welding closed loop sensor response times of 1 to $10 \mathrm{msec}$ have been identified by researchers working in this field. Closed loop sensor response time is defined as the time required for sensor information to be sent from the sensor to the control platform, evaluated for system response and the response effected at the point of process. Typical mechanical systems of the size and configuration of robots that can be used for welding have incorporated sensor update rates of 10 to 20 updates per second. This corresponds to a 50 to $100 \mathrm{msec}$ closed loop response time for sensor input. A sensor response may effect a power change, a path modification, a change in process depth via relocation of the laser focal point or tungsten tip, or a gating of the process power. This real-time parameter change would then effect the next planned sequential operation which would require a response to keep the system on the original plan. It can immediately be seen that this process could easily degenerate into a pattern of chaos. The execution of a planned path using sensor input to effect repair and provide continuous process flow is the problem identified in this project.

\section{Approach Incorporating Precision Control}

The problem identified above has numerous levels of complexity associated with it depending on the process selected for research and development. Examples of this are seen when looking at the differences between the two identified processes, laser cutting and welding. In these two cases, the process delivery mechanism and the energy source are identical. Both use a motion delivery platform such as a robotic arm and a high powered fiber optic delivered laser beam to accomplish the task. Cutting, which is identified here as the less difficult of the two processes, requires only the controlled removal or ablation of selected material. Welding, which is considered more difficult, requires the controlled fusion of two separate materials into one. Welding also requires a specific outcome of predetermined parameters such as joint width, penetration depth, and heat effected zone. Both have common fundamentals that can be pursued in parallel in the 
early stages of a research and development program. These are positional control of the focal point, power adjustment, and focal point speed relative to the work being processed.

The approach taken in this research and development program was to first realize and demonstrate the ability to affect process parameter modification in real-time while delivering the laser cutting process, and then extend it to the welding process where more specific sensors can been incorporated as they are identified or developed.

The first step of autonomous control of the laser cutting process is the computer generation of accurate robotic path plans. These are used to establish the baseline motion for the cutting process that is desired. The paths are generated using the proven technologies developed in Sandia National Laboratories' Intelligent Systems and Robotics Center where extensive work on computer path planning and execution have been performed. After generating accurate and collision free paths, these paths are used to execute the process under sensor based control. The sensors provide information via a number of communications modes to the robotic controller where a real-time path modification module resides. This module uses the sensor information to provide offsets to the path being executed in real-time. For the case of laser cutting, the critical parameters identified are focal point location, and the power output of the laser. The sensors identified for control of the parameters noted are capacitive sensing, and Laser Induced Breakdown Spectroscopy (LIBS) respectively. As the computer generated path plan is executed, the real-time path modification module will continually drive the robot back towards the original planned path with the sensor input providing offsets for optimal process performance.

In this program, the combination of extremely precise robotic hardware, advanced path planning and execution software, real-time path modification capability, and effective sensors will provide the platform for advanced research and development into real-time sensor based control of non-contact processes such as laser cutting and welding. The sections that follow outline the software and hardware incorporated into the realization of this platform. 


\section{Software}

This section describes the software used in the development of the robotic platform for this program. The configuration of the software components was performed with the goal of creating a path that could reliably be executed using CAD models as the primary information source. Speed of communication between software modules and compatibility of shared data sets was of primary concern. Figure 1 shows the logic diagram of the supervisory control platform and the communications mode between it and the other controllers used in the scheme. It also identified the required software modules and their means of communications.

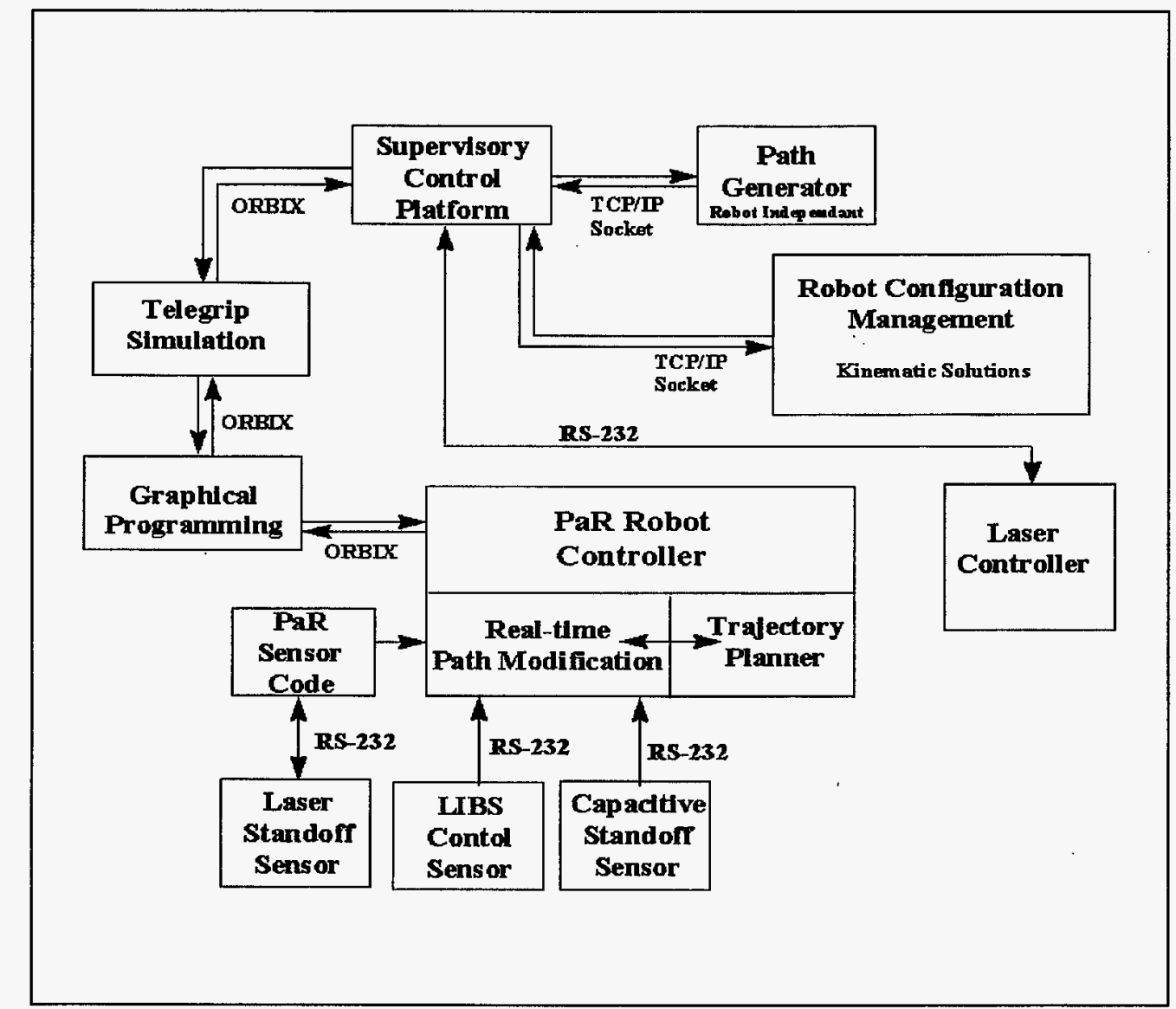

FIGURE 1. LOGIC DIAGRAM OF PROJECT SOFTWARE CONTROL

As shown, the supervisory control platform communicates with the path generation module and the robot configuration management module via TCP/IP sockets.

Communications between other modules is via ORBIX, and specific communication between the graphical programming module and the PaR robotic controller is via Local Area Network Distributed Supervisory Control And Programming Environment, or LandScape. The balance of the communications is via standard RS-232 lines. This 
configuration allows for the fastest and most effective means of handling the large amounts of information associated with graphical programming files and graphical information processing. This software configuration provides the means to create a base path for the robot to execute under sensor based control and is the fundamental technology needed for bringing real-time process control to the laser welding/cutting process. The following is a brief description of the software components incorporated into this system.

\section{Robotic Path Planning}

This software module is responsible for the generation of tag points that are used to generate robotic paths in the next software module. It uses CAD models of the parts and robotic workcell to accomplish this. The points are generated using the $\mathrm{CAD}$ models with special feature extraction capability. This technology is robot independent. The output is a file of tag points that the control platform provides to the Robotic Configuration Management software module for use in creating robotic configurations in joint mode.

\section{Robotic Configuration Management}

This software module generates robot kinematic solutions in joint configuration. The input is the file of tag points generated in the previous module and the output is a kinematic solution in robot joint configuration. The robot configuration module checks for and excludes singularities ${ }^{3}$ in the execution of the robotic path. It also assures that the start sequence configuration of the robot is correct to exclude joint limit problems while executing the path.

\section{Graphical Simulation}

The input for this module is the joint values generated in the Robotic Configuration Management module and the output is a simulation of the motion generated for the Graphical Programming module. This module uses the tag point generated joint values passed to it from the robot configuration management module to simulate the path before execution. This simulation highlights collisions and near misses of the robot with features in the workcell. This allows for a complete visualization of the path planned for execution before it is passed to the graphical programming module for final path code generation. The operator is given a visual verification and an decision point to proceed with process in this step.

\section{Graphical Programming}

The input for this module are the paths generated in the Graphical Simulation Module after they are verified to be correct. This module converts the joint values to path sequences and applies a generic approach and depart path for the robot to assure that the path being executed does not contain plans that pass through joint limits. This module 
also tracks the path as it is being executed using graphical simulation and creates a highlighted line of the executed tool tip path on the part model. 


\section{Hardware}

This section describes the hardware used in this project. The hardware configuration was established to meet the needs established in the approach to the problem outlined in the previous sections. Figure 2 details the hardware layout and the communications scheme that was created to assure that the sensors and hardware interacted effectively and at the highest possible data transfer rate. The sections that follow detail the hardware chosen and their contribution to the project.

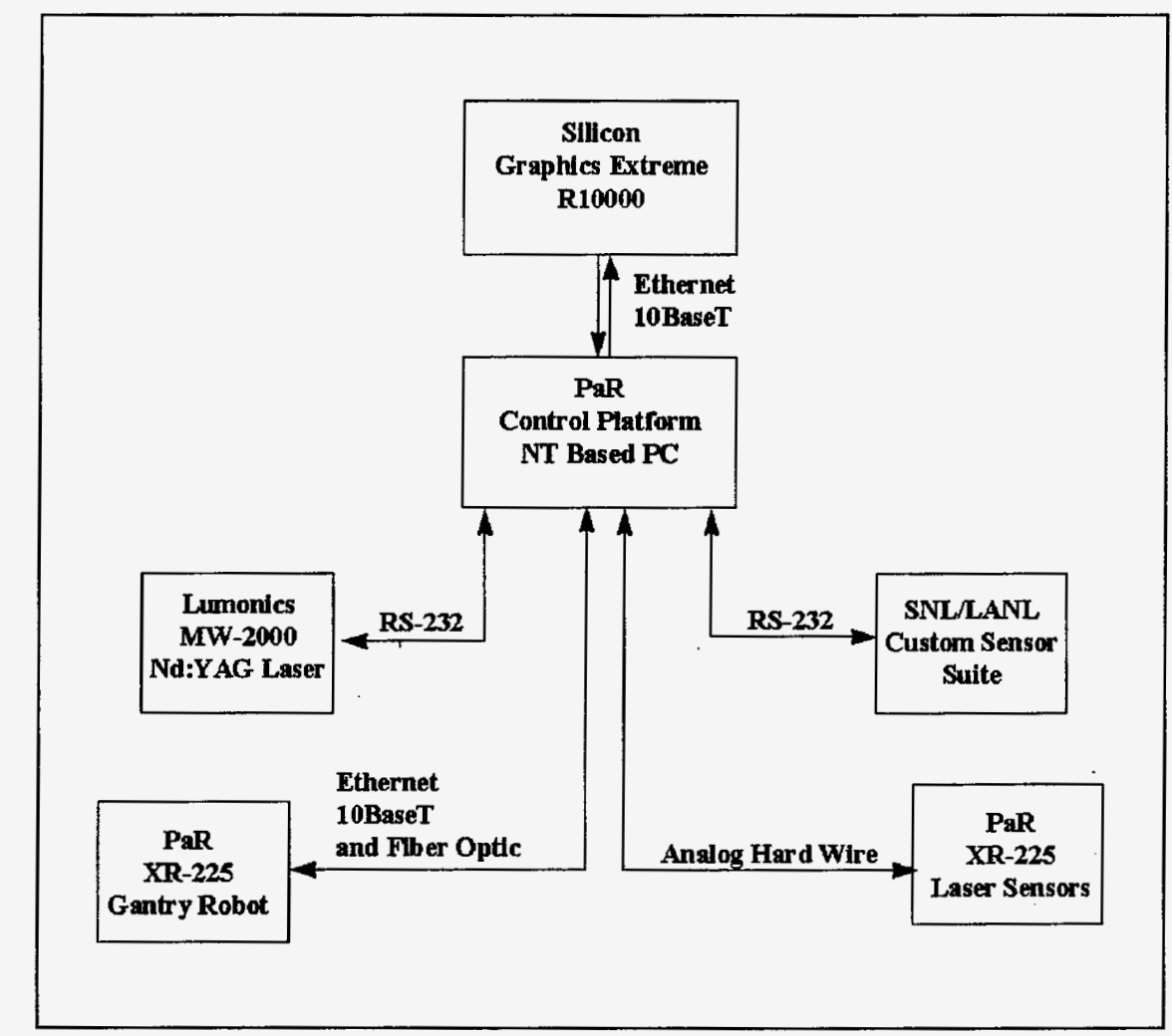

Figure 2. HARDWARE DIAGRAM WITH COMMUNICATION TECHNOLOGY IDENTIFIED

\section{Robotic Platform}

The XR 225 overhead gantry robot is an extended-reach industrial robot that provides a large work envelope and high precision accuracy for large scale process operations. The robot is runway-mounted and will provide five degrees of freedom (axes): three translation movements $(\mathrm{X}, \mathrm{Y}, \mathrm{Z})$ : and two rotational movements, main rotation (yaw or Theta 1) and pivot (Pitch or Theta 2). The runway is mounted on a support structure. 
The XR 225 robots are all electric servo motor driven and microprocessor controlled from the PaR CIMROC $4000 X$ controller. The XR 225 utilizes absolute and independent position feedback systems on all axes. These systems measure the absolute position of the axes and therefore require no homing upon start-up or loss of power. Additional position feedback sensors are mounted at the output of each axis eliminating position errors caused by gear train wear, inaccuracies, and compliance. Figure 3 summarizes the performance and characteristics for each of the axes on the XR 225.

\begin{tabular}{|c|c|c|c|c|c|c|c|c|c|c|}
\hline \multicolumn{11}{|c|}{ XR 225} \\
\hline \multicolumn{11}{|c|}{ Performance Characteristics } \\
\hline $\begin{array}{l}\text { Atis } \\
\text { Nitinber }\end{array}$ & Direction & $\begin{array}{c}\text { Travels } \\
\text { inches desreess }\end{array}$ & $\begin{array}{c}\text { Min. } \\
\text { Speed } \\
\text { ips (radps) }\end{array}$ & $\begin{array}{c}\text { Max. } \\
\text { Speed } \\
\text { ips (radps) }\end{array}$ & $\begin{array}{c} \pm \\
\text { Accuracy } \\
\text { in (arc sec) }\end{array}$ & \begin{tabular}{|c|} 
\\
Reperatailitity \\
in (arc sec)
\end{tabular} & $\begin{array}{l}\text { Resolution } \\
\text { in (arc sec) }\end{array}$ & $\begin{array}{l}\text { Capanity } \\
*(i n=*)\end{array}$ & Drive Mechanism & Position Feedhack \\
\hline \begin{tabular}{|c|}
$\mathrm{X}$ \\
$J 1,57$ \\
\end{tabular} & Longitudual & 133.00 & 0.024 & 40 & 0.003 & \begin{tabular}{|c|}
0.001 \\
\end{tabular} & 0.00014 & $600^{4}$ & $\begin{array}{c}\text { Dual drive } \\
\text { Rack and Pinion }\end{array}$ & $\begin{array}{l}\text { Absolute } 21 \text { bit } \\
\text { Dual Resolvers }\end{array}$ \\
\hline $\begin{array}{l}\mathrm{Y} \\
\mathrm{J} 2\end{array}$ & Lateral & 108.70 & 0.024 & 40 & 0.003 & 0.001 & 0.00014 & $600^{4}$ & Rack and Pinion & $\begin{array}{l}\text { Absolute } 21 \text { bit } \\
\text { Dual Resolvers }\end{array}$ \\
\hline $\begin{array}{l}\mathrm{Z} \\
J 3\end{array}$ & Vertical & 72.00 & 0.008 & 14 & 0.003 & 0.001 & 0.00007 & $1000^{5}$ & Ball Screw & \begin{tabular}{|l||} 
Absolute 21 bit \\
Dual Resolvers
\end{tabular} \\
\hline $\begin{array}{l}\mathrm{C} \\
\mathrm{J} 4\end{array}$ & Yaw & (370) & $(0.001)$ & (2) & (15) & (3) & (1.35) & $\left(7500^{6}\right)$ & Sumitomo Cycloidal & \begin{tabular}{|l||} 
Absolute 21 bit \\
Dual Resolvers
\end{tabular} \\
\hline $\begin{array}{c}\mathrm{B} \\
15 \\
\end{array}$ & Pitch & (210) & $(0.001)$ & (2) & (15) & (3) & $(1.35)$ & $\left(9000^{6}\right)$ & \begin{tabular}{c|} 
Timing belt \\
Sumitomo Cycloidal \\
\end{tabular} & $\begin{array}{l}\text { Absolute 21 bit } \\
\text { Dual Resolvers }\end{array}$ \\
\hline \multicolumn{11}{|l|}{ Notes: } \\
\hline
\end{tabular}

FIGURE 3. PAR ROBOTIC SYSTEM ACCURACY BY AXIS

To accomplish the high degree of accuracy on the gantry robot system, the XR runway beams, bridge beams, and mast linear way seats are precision machined at approximately one half the machining tolerances of the standard system. Heidenhain Linear scales were used on all linear axes. This device has a measuring accuracy of 0.0002 inches and resolution of 0.00004 (with an electronic interpolation unit). Heidenhain Rotary encoders were used on yaw and pitch axes. This device has a 5 arc second accuracy and 0.72 arc second resolution (with an electronic interpolation unit). These Heidenhain scales and encoders have a semi-absolute feature which allows the axes to be homed after moving only a small distance $-100 \mathrm{~mm}$ for linear axes and 20 degrees for rotary axes. These devices provide higher precision feedback information which enables greater mechanical error correction (MEC) using the existing PaR software.

A CIMROC 4000x Robot Controller provides the system with a complete range of motion controls, including multiple coordinate systems, straight-line tool tip motion, timeoptimized interpolation, circular interpolation and relative moves (point transformations). This controller incorporates advanced data processing capabilities, user-defined interrupts, interrupt processing, analog or digital $\mathrm{I} / \mathrm{O}$, and serial communications. CIMROC 4000x open control architecture is based on modular, distributed processor design. This design enables separate, simultaneous control of supervisory, trajectory, and servo functions. It 
also provides maximum flexibility in hardware to integrate advanced, real-time motion control options, including intelligent interfaces for welding, force-tactile, vision and seamtracking packages. A photo of the PaR gantry robotic system is shown in Figure 4. The installation of the system included a Lumonics MW-2000 Nd:YAG laser and supporting safety systems such as laser interlocks at doors and window shielding.

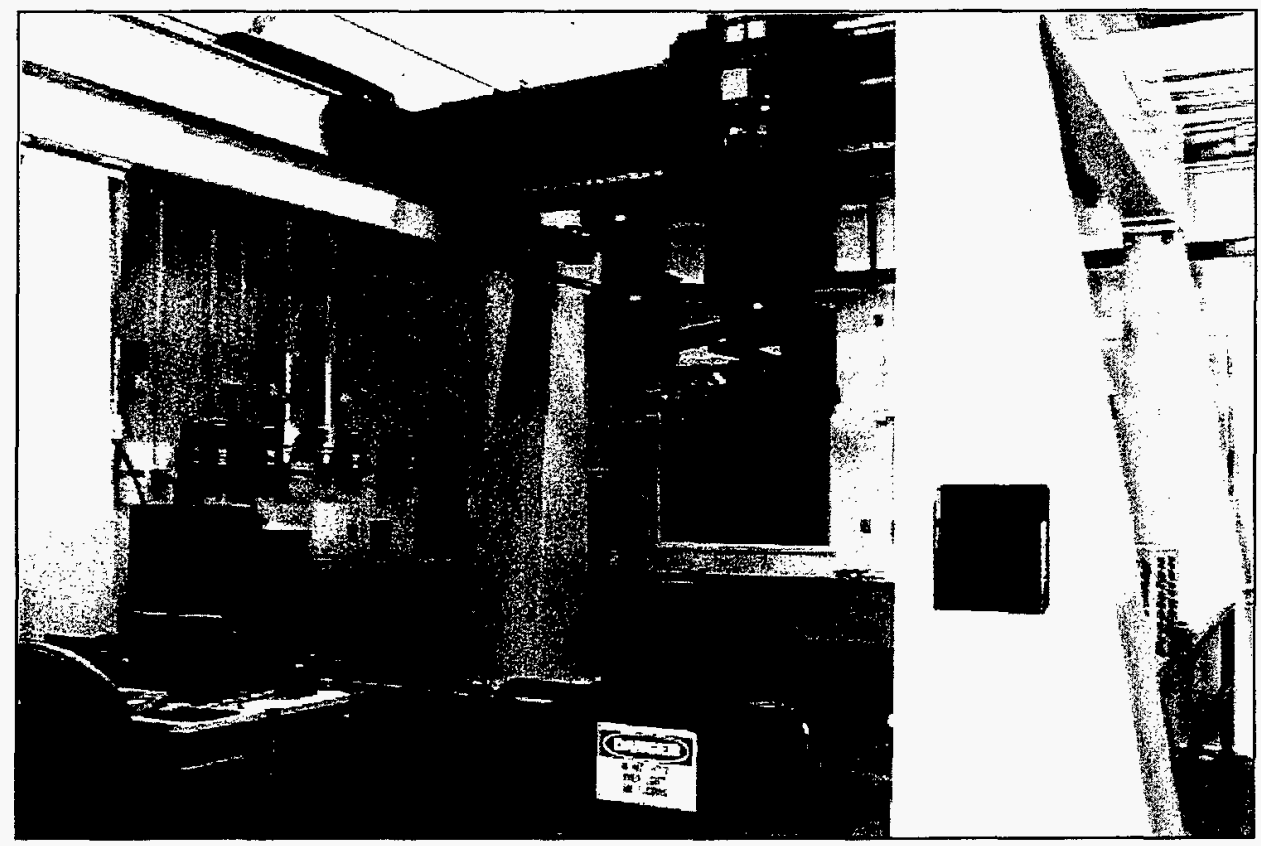

FiguRe 4. Photo of the PAR XR-225 Precision Robotic Gantry Platform

The control system supervisor is a high speed 32 bit processor and floating point coprocessor residing on a passive ISA (Industry Standard Architecture) backplane. It supports standard hardware options such as various graphics display adapters, hard disk and tape storage, extended memory, various LAN products, multiple intelligent and nonintelligent serial ports and other third-party hardware. Sophisticated software packages are available which significantly expand system capabilities, including CAD/CAM, local area networks and other host communications, data base management, word processing and graphics.

The controller can be programmed using an operating system (PC-DOS ${ }^{\oplus}$ ) that provides an interface for creating robot tasks using standard Microsoft $\mathrm{C}$ language. This high-level language in conjunction with $\mathrm{PaR}$ library products provides users with all the development tools needed to undertake advanced robot control applications. Furthermore, any PC computer, or true compatible, can be used as a remote workstation for developing programs. 
A 32 bit trajectory $\mathrm{CPU} /$ math coprocessor interprets position commands and performs mathematical computations for simultaneous coordination of multiple robot axes with controlled acceleration and velocity. A 32 bit Digital Signal Processor (DSP) servo processor, executes the interpolated position commands sent by the trajectory to effect robot motion while simultaneously monitoring axis performance, minimizing dynamic positioning errors. Real Time Path Modification (RPM ${ }^{\mathrm{TM}}$ ) coprocessors execute in parallel with the trajectory processor performing path modification and process control for specific applications.

RPM executes in the controller with robot and interfaces to customer supplied sensors and computers. RPM enables real-time robot path modification based on input from an external computer or a variety of sensors. Its primary purpose is for contouring and adaptive control. The RPM package can supply actual robot position information, in a variety of interfaces and data formats, to acquisition or other external computers.

The hardware consists of a single board computer residing within the CIMROC $4000 \mathrm{x}$ motion control system. It communicates path modification information and receives robot position information from the motion control system and receives biasing commands from and communicates actual robot position to an external computer.

The RPM module accepts biases in joint, tool, or base coordinate frames. It receives path bias data from the sensor, checks and limits it, and passes the resultant data to the motion control system to bias the programmed path of the robot. The bias remains in effect until new data is received. The biases are applied to the position servo system at an update rate of $100 \mathrm{HZ}$ for CIMROC 4000x controllers. The data is communicated in a compact ASCII string or binary packet format over an RS-232 or optional RS-422 serial port at up to 19200 baud. The sensor used must be intelligent enough to provide this data in a format acceptable to RPM.

The RPM module also supplies the robot's actual position in joint or base coordinate frames through a serial interface. This serial data is available in a variety of compact ASCII string or binary packet formats at a programmable rate up to 100 updates per second. This real-time position information is often used to position-stamp data collected during data acquisition operations such as robotic radiographic or ultrasonic inspection. Other applications include real-time force control, real-time vision-based tracking, and real-time remote graphic rendering of robotic cell operations.

\section{Sensors}

The program originally identified two sensor technologies for the sensor based control effort. These were capacitive standoff sensing ${ }^{4}$ and spectroscopy ${ }^{2}$. The capacitive standoff sensing technology became the primary thrust in the initial phase of the program because of the time required to develop and test the spectroscopic sensor. The standoff sensor was installed on the robotic platform and testing and calibration was started. A 
description of the capacitive standoff sensor follows, but a more detailed description can be found in reference 3 of this report.

The Sandia developed capacitive sensing technology has been directed at the development of a Standoff Sensor System used for the control of robotically deployed tools. These tools are utilized primarily in the Department of Energy's Decontamination and Dismantlement (D\&D) activities. The sensor system combines four individual sensor elements to provide non-contact, multiple degree-of-freedom control of tools at distances up to five inches from a surface. This sensor has previously been successfully integrated to a metal cutting router and a pyrometer, and utilized for real-time control of these tools. Experiments demonstrate that the system can locate stationary surfaces with a repeatability of 0.034 millimeters. This capability was extended to the robotic platform in this program to accomplish the same tasks but under faster update requirements.

The standoff sensor consists of a 100 millimeter diameter capacitive sensor that is encapsulated inside a rugged plastic housing producing a light-weight assembly designed to support a variety of robotically deployed tools. Figure 5 shows the sensor and deployment configuration on the robotic platform. The sensor consists of four individual elements whose output can be combined in a variety of ways to control the critical degrees-of-freedom of the tool. Typically, the three degrees-of-freedom controlled are the distance of the tool from the surface, and the two orientations required to keep the tool normal to the surface.

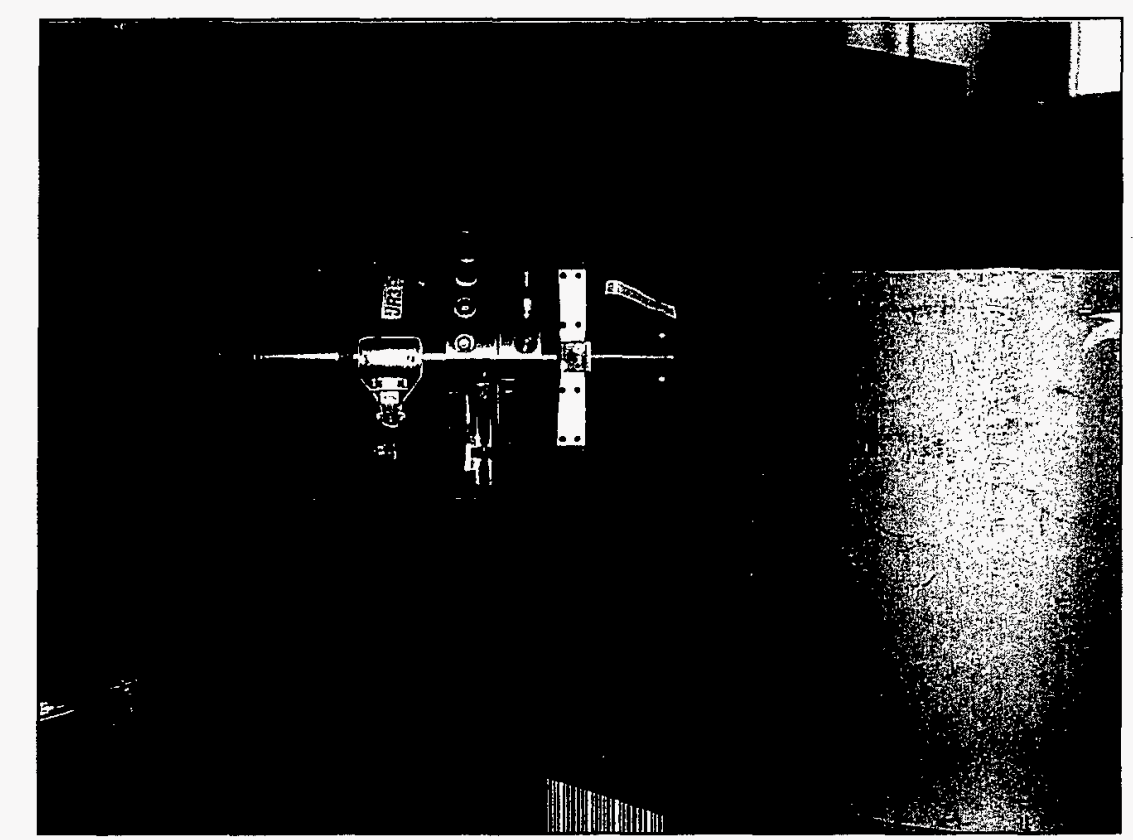

Figure 5. CAPACITIVE STANDOFf SENSOR ON RoBotic Platform

The standoff sensor is fabricated on a printed circuit board with four sets of electrodes on the sensor as shown in Figure 6. Each set of electrodes consists of an oscillator and a receiver. The oscillator electrode is driven by a low voltage sinusoidal signal in the $100 \mathrm{~Hz}$ 
range. This signal is coupled through the space in front of the sensor to the receiver electrode to produce an electric field which extends outward from the sensor face. The receiver electrode is connected to a charge amplifier stage for sensing capacitor charge. The output of the sensor is a signal of the same frequency as the oscillator, but reduced in magnitude according to the capacitance between the electrodes. Any objects or obstacles near the sensor will change the capacitance between the electrodes and result in a corresponding change in the amplitude of the sensor output signal. These amplitude changes are detected and converted by the signal conditioning electronics described in subsequent paragraphs.

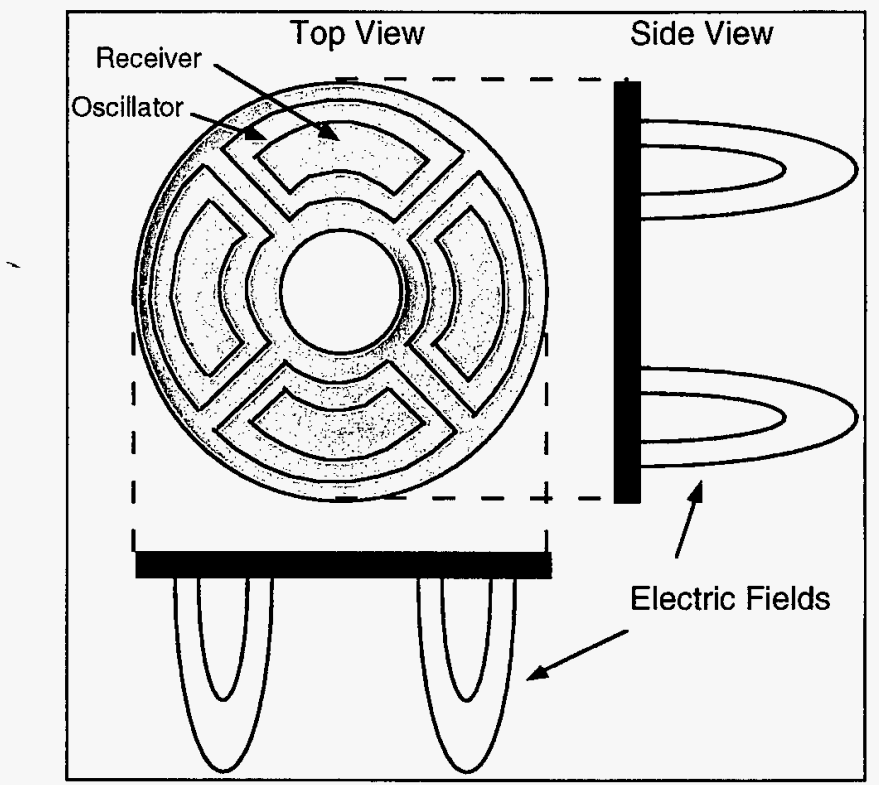

FIGURE 6. DIAGRAM OF STANDOFF SENSOR

The sensor system has four channels of output, one for each electrode pair. Each channel is a signal proportional to the distance from one electrode pair to the surface of a workpiece. The four electrode pairs on the sensor face give it functionality for both standoff and orientation control. Because the sensors are fabricated on ordinary printed circuit boards, they are economical to produce and can be made larger or smaller for different applications. This unique capacitive sensing technology is not affected by stray capacitances to ground (such as between the sensor and workpiece) and is insensitive to the electric potential of the workpiece. As a result, both conductive and nonconductive workpieces can be detected. Also, because the electric fields produced by the sensor can penetrate dielectric materials, the sensor can be protected from the environment by a plastic or epoxy covering. For this deployment, the Standoff Sensor is encapsulated inside a dense plastic $\left(\right.$ Delrin $\left.^{\oplus}\right)$ housing with a 6.4 millimeter wall thickness.

Signal conditioning electronics convert the sensor signals into analog voltages to provide useful information about workpiece proximity. The analog signal processing consists of a mixer stage, a synchronous demodulator stage, and an output gain/filter stage. These electronics measure the small amplitude changes in the $100 \mathrm{kHz}$ sensor signal caused by changes in capacitance between the sensor electrodes. The synchronous demodulator stage 
rejects noise and signals of other frequencies. Sensor signals can be processed at different gain settings to produce various distance detection ranges. These detection ranges are made accessible through discrete gain jumper settings within the signal conditioning electronics. In addition, the output of an individual sensor element can be simultaneously processed through multiple signal processing channels at different gain settings to produce multiple detection ranges at the same time. Each sensor signal is simultaneously processed through two signal processing channels. Thus, the four sensor elements on the Standoff Sensor result in eight channels of output from the signal conditioning electronics. The output range of all signals is \pm 10 volts.

The signal conditioning electronics as described can be located up to 15 meters from the sensor. For remote sensing applications cable runs longer than 15 meters are required. To solve this problem, an embedded computer was integrated with the signal conditioning electronics to convert the sensor signals into a serial data stream. The output voltage signals from the analog electronics board are digitized by the computer and converted into a serial data stream suitable for transmission via an RS-485 or other network. The electronics package is compact enough that it can be placed at the end effector of the robot or in an enclosure at the robot controller. The serial data stream can then be interpreted by the robot controller and used to control the position and orientation of the robot arm.

\section{Control Platform}

The platform chosen for the supervisory control of the system was Silicon Graphics Extreme with ATM communications capability. This platform carried the entire path planning and execution software as well as the simulation modules. The system communicates through Ethernet links to the PaR robotic system. The Ethernet communications are done over 10-Base-T and fiber optics lines. The Silicon Graphics platform was chosen because of its capabilities to support the graphical control and simulations software requirements necessary for path planning and execution. 


\section{Results}

The principal deliverable of this effort was a real-time sensor based controlled robotics platform that incorporates $\mathrm{CAD}$ model based path planning and execution, and graphical programming technologies. This system was demonstrated and tested to the extent that time and funding allowed.

The path planning and motion execution development using simulation technology and Sandia developed path planning and collision avoidance software was deemed successful. A full demonstration of this capability was realized. This demonstration started with the placement of an 18 in. diameter stainless steel tube in the robotic work cell. A CAD model of the tube was built and transferred into the world model using Deneb Telegrip ${ }^{\circledR}$ simulation software. A simulation frame of the stainless tube in the workcell is shown in Figure 7.

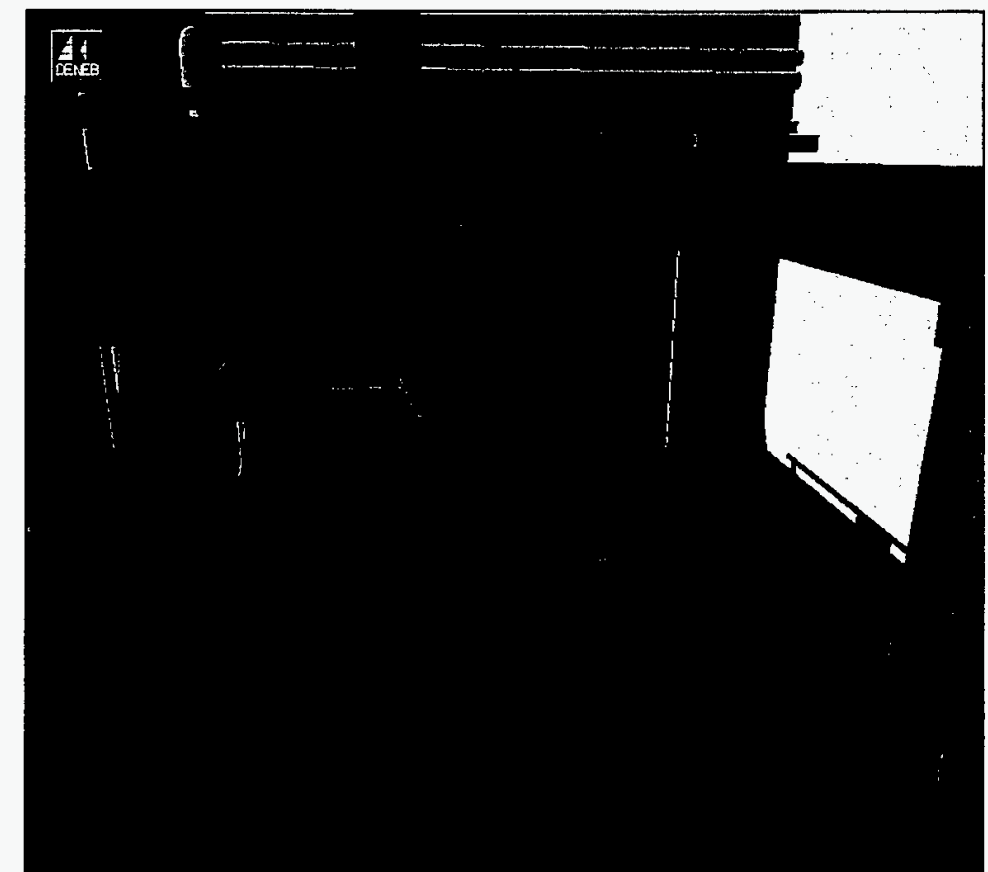

FigURE 7. SimULATION OF ROBOTIC PlatToRM AND WORKCELL

The world model was then viewed and accepted by the operator. The operator was then allowed to create a cutting path by moving an intersecting plane in the tube. This is shown in a simulation frame in Figure 8. This intersecting plane placement established the cut that was to be performed by the laser. The points created by the intersection of the plane with the tube were then used to create tag points along the tube. These tag points were then used to create a path in robotic joint configuration. This was performed by the Robotic Configuration Management software module. The path generated is shown in 


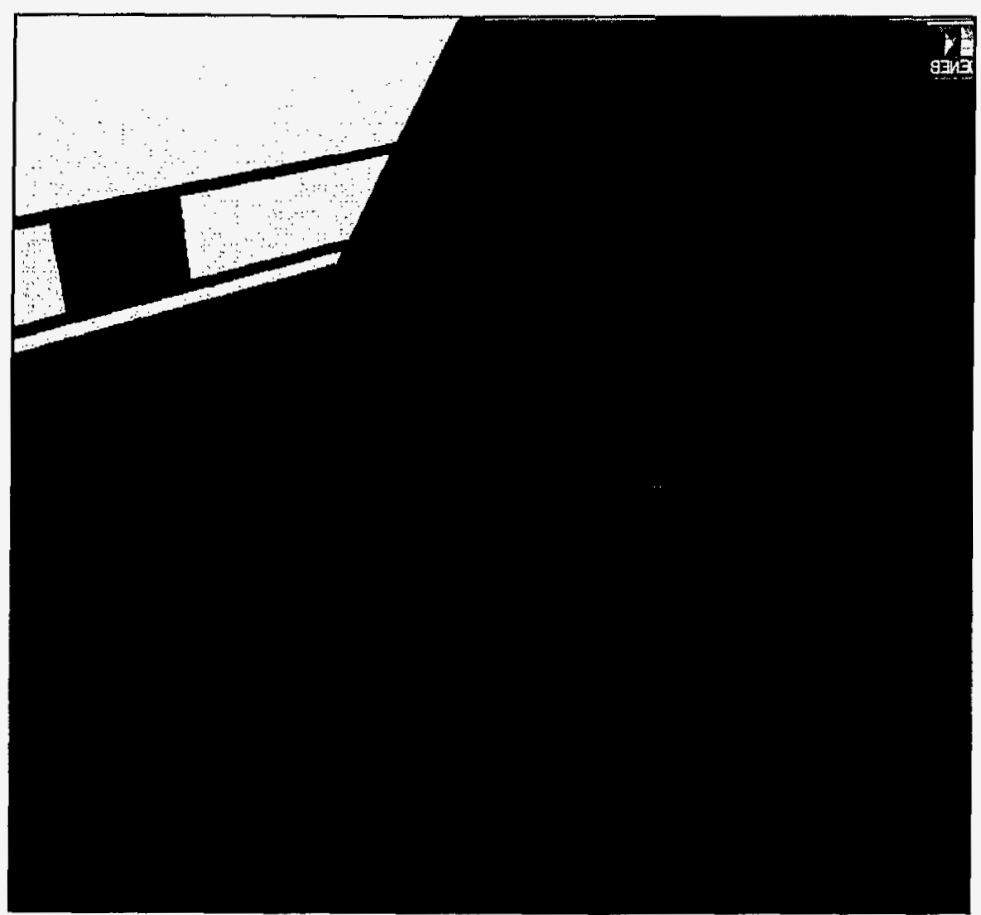

Figure 8. Simulation of Planar Cut Through Cylinder To Establish Path

Figure 9. This path was then checked to assure that it could be executed without singularities. At this point, the path planning and configuration of the robot have been generated and stored as points and joint values that could be executed on the robot platform.

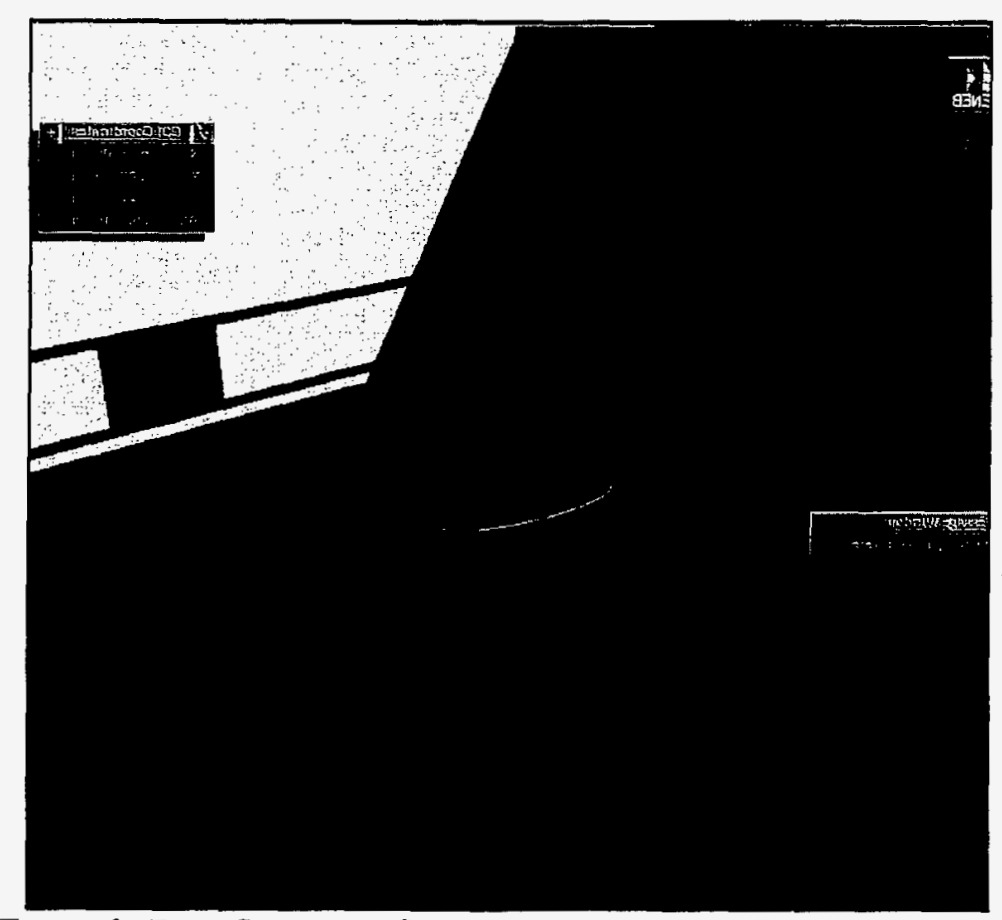

FigURE 9. PATH SHOWN ON CYLINDER 
The next step in generating the path is the verification that it is collision free during execution. This assures that the robotic arm and any moving components of the robotic platform do not collide with tools, fixtures or the work piece itself while executing the path. To accomplish this, the joint values generated in the last step were loaded into the graphical programming module. In this module the joint values are used to generate a robotic path plan including a generic approach and depart from the work location. This assures that the robotic arm can execute the path without exceeding joint limitations and that it is collision free. If a collision exists, the collision point is highlighted in red and a re-planning effort is performed. A simulation frame of a highlighted collision is shown in Figure 10. The planning process is performed under the supervision of the operator and a verification to execute the plan using the robotic platform is provided to him. Once a path has been generated that is acceptable, the operator starts a downloading process.

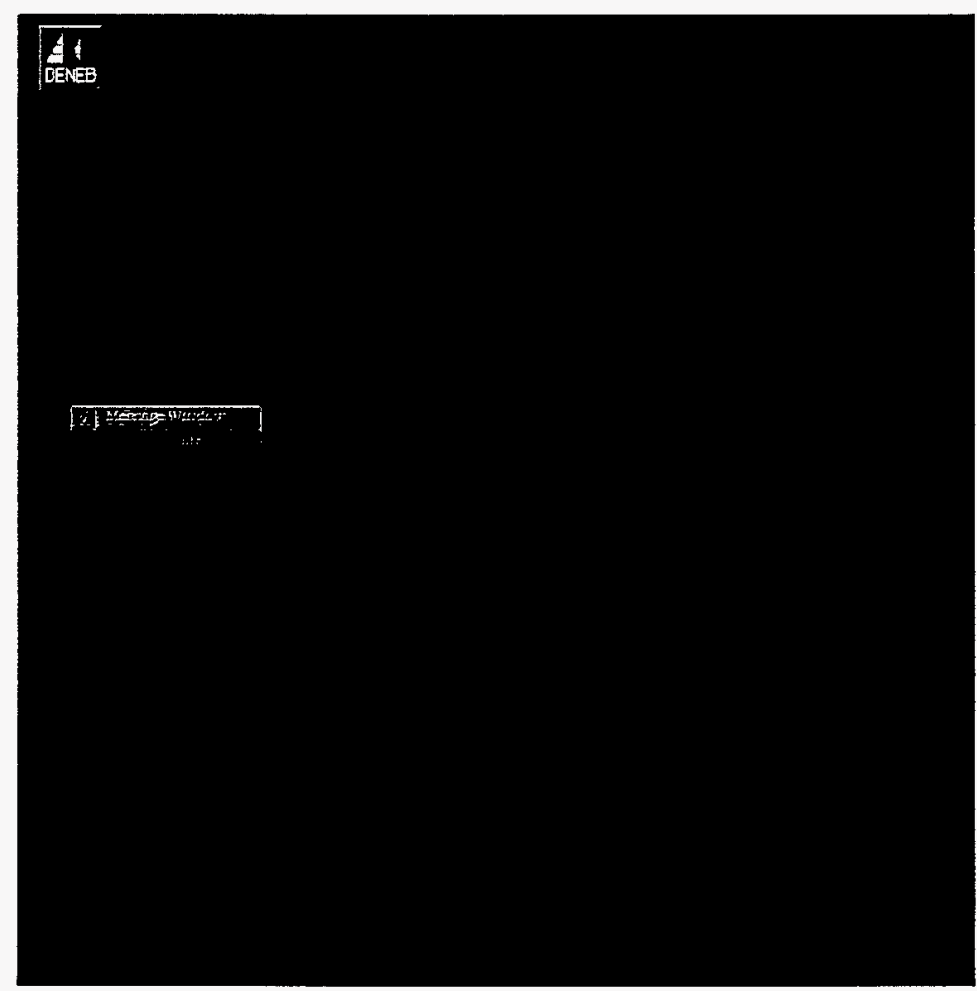

FigURE 10. SIMULATION SHOWING A COLLISION HIGHLIGHTED

The path is downloaded to the robotic control platform using Landscape, a CORBA ${ }^{\circledast}$ based interface that was specified by Sandia and implemented onto the Cimroc 4000X controller by PaR Systems. At this point the robotic platform executes the planned motion. Figure 11 shows a frame of what the operator sees at the execution of the path in the simulation step of the process. In the simulation step, not only is the robot motion shown, but the path that is being executed at the tool tip is also provided.

The development and implementation of sensor based control was also accomplished. The use of sensors to effect path modification was realized with the installation and 
testing of the capacitive sensor on the platform. A higher speed version of the current technology was designed and tested. At the closure of the project in FY97, the generic

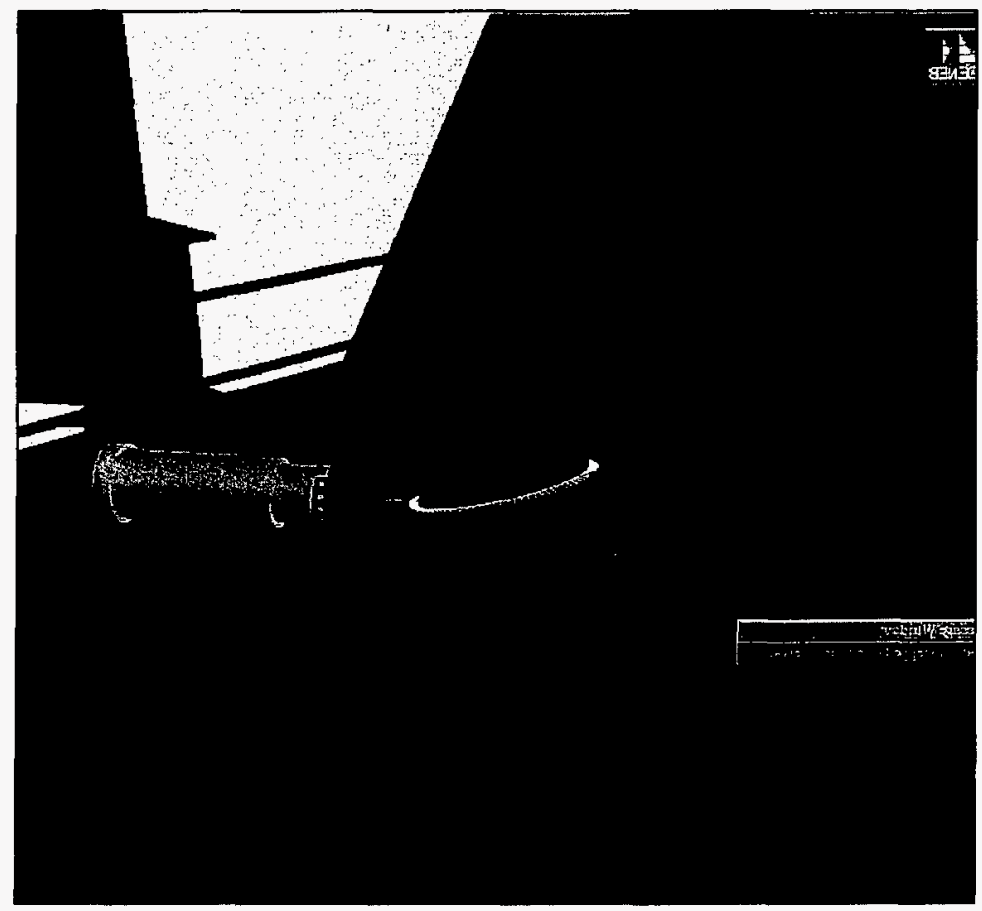

Figure 11. Simulation OF RoBot ExeCUTING PlanNED PATH

capacitive standoff sensor had been mounted on the platform and calibration started. A more application specific standoff sensor had been designed and scheduled for implementation and testing. The results from the calibration and testing of the generic sensor showed that the system was capable of providing the necessary real-time path modification to a computer generated and executed plan. A typical example of the capability provided by this sensor is the ability of the sensor to hold the robotic end effector a given distance from the cylinder used in this demonstration. This cylinder was out of round by as much as .200 of an inch and if the path were executed without sensor control, the focal point of the laser would have been mis-positioned by that amount causing the process to fail.

A second development in the real-time sensor based control effort was the redesign and installation of the Real-time Path Modification capability. PaR Systems, Inc. delivered a four fold increase in path modification speed under sensor input. The closed loop sensor response time was decreased from $37 \mathrm{msec}$ to $10 \mathrm{msec}$ with this design change. This was accomplished by redesigning the RPM controller boards using higher speed multiple coprocessors and custom written communications algorithms. This accomplishment moved the update rate from 25 per second to 100 per second. This robotic platform is now one of the fastest integrated systems platforms built. 
In summary, the robotic platform generated under this program has the ability to create and execute precision paths using state-of-the-art path planning and graphical programming technologies. After the path is generated and ready for execution, the platform provides a means to modify and update those paths under real-time sensor based control.

This platform provides a precision test bed for the development of the sensors required for the real-time autonomous control of non-contact processes such as laser cutting and welding, laser sintering of powdered metal, plasma coating, and high speed plasma cutting. It is also a test bed for the development of software capability that support realtime process control. 


\section{References}

${ }^{1}$ Pamela K. Benicewicz and D. Kirk Veirs, "Characterization of Gadolinium via the Temporal and Spatial Evolution of Emissions from Laser-Created Plasmas", submitted for publication, Los Alamos National Laboratory, Los Alamos, NM. September, 1997.

${ }^{2}$ D. Schmitt, J. Novak, J. Akins, "Capacitive Sensor for High Resolution Weld Seam Tracking", Sandia Report SAND95-0885 UC-706, Unlimited Release, Sandia National Laboratories, Albuquerque, NM, May, 1995.

${ }^{3}$ J. Craig, "Introduction To Robotics: Mechanics and Control" $2^{\text {nd }}$ Edition, AddisonWesley Publishing Company, Reading, MA, 1986, 1989. A definition of singularities is found here with further references.

${ }^{4}$ D. Schmitt, T. Weber, J.C. Lui, "Capacitive Tool Standoff Sensor For Dismantlement Tasks", American Nuclear Society Conference, Augusta, GA, April 27 -May 1, 1997. 


\section{Distribution:}

$\begin{array}{lll}1 & \text { MS-0188 } & \text { LDRD Office, 4523 } \\ 1 & \text { MS-1002 } & \text { Pat Eicker, 9600 } \\ 1 & \text { MS-1006 } & \text { Pablo Garcia, 9671 } \\ 1 & \text { MS-1007 } & \text { Al Jones, 9672 } \\ 6 & \text { MS-1007 } & \text { Randy McKee, 9672 } \\ 1 & \text { MS-1006 } & \text { Lilita Meirans, 9671 } \\ 1 & \text { MS-1003 } & \text { Dan Schmitt, 9611 } \\ 1 & \text { MS-1010 } & \text { Dan Small, 9622 } \\ 1 & \text { MS-1008 } & \text { Peter Watterberg, 9621 } \\ 6 & \text { MS-1008 } & \text { Sharon Blauwkamp, 9621 } \\ 1 & \text { MS-9018 } & \text { Central Technical Files, 8940-2 } \\ 2 & \text { MS-0899 } & \text { Technical Library, 4916 } \\ 2 & \text { MS-0619 } & \text { Review \& Approval Desk, 12690 } \\ & & \text { For DOE/OSTI }\end{array}$




\section{M98004415 \\ |||||||||||||||||||||||||||||||||||||||||||||||||||}

Report Number (14)SAND-98-0702

Publ. Date (11) $\frac{1996023}{\text { Sponsor Code (18) }}$
UC Category (19) 